\title{
New Exact Solutions for the Maccari System
}

\section{Abdelrahman $\mathrm{MAE}^{1 *}$ and Hassan $\mathrm{SZ}^{2}$}

${ }^{1}$ Department of Mathematics, Faculty of Science, Mansoura University, Egypt

${ }^{2}$ Department of Mathematics, College of Education, Imam Abdulrahman Bin Faisal University, Saudi Arabia

\begin{abstract}
In this article we apply three mathematical methods for solving the Maccari system, namely, the $\exp (-\varphi(\xi))$ expansion method, the sine-cosine approach and the Riccati-Bernoulli sub-ODE method. These methods are used to construct new and general exact periodic and soliton solutions of the Maccari system. This nonlinear system can be turned into another nonlinear ordinary differential equation by suitable transformation. It is shown that the $\exp (-\varphi(\xi))$-expansion method, the sine-cosine method and the Riccati-Bernoulli sub-ODE method provide a powerful mathematical tool for solving a great many systems of nonlinear partial differential equations in mathematical physics.
\end{abstract}

Keywords: $\operatorname{Exp}(-\varphi(\xi))$-expansion method; Sine-cosine method; The Riccati-Bernoulli sub-ODE method; Maccari system; Solitary wave solutions

\section{Introduction}

Nonlinear wave phenomenon play a major role in many natural sciences including mathematics, biology, and particularly in many branches of physics, such as nonlinear optics, condensed matter physics, plasma physics, chemical physics, solid-state physics, fluid dynamics, [1-7]. Actually, there are so many interesting applications of the previous natural sciences like the schrodinger equation, the Phi-4 equation, the 2D Ginzburg-Landau equation, the generalizedZakharov system, the model of blood flow in arteries, the diffusive predator-prey system, shallow water equations, etc.

According to the import role of these equations, various papers interested in finding solutions of them even numerically or analytically. These solutions might be essential and important for the exploring some physical phenomena. Therefore investigating new technique for deterministic case to solve more complicated problems. Thus, many new methods have been proposed, like as the homogeneous balance method [8,9], the F-expansion method [10-12], the tanh-sech method [13-15], the Riccati-Bernoulli sub-ODE method [16,17], Jacobi elliptic function method [18,19], sine-cosine method [20-22], extended tanhmethod [23-25], $\left(\frac{G^{\prime}}{G}\right)$-expansion method [26,27], and exp-function method [28-30].

The objective of this paper is to apply three mathematical methods, specially, the $\exp (-\varphi(\xi))$-expansion method, the sine-cosine method and the Riccati-Bernoulli sub-ODE method, for finding various new exact solutions of the Maccari system. This system is extremely presented to define the motion of the isolated waves, localized in a very small part of space, in many fields such as hydrodynamics, optics, quantum field theory. Moreover it used in plasma physics to describe the behavior of the sonic Langmuir solitons. We give new solutions and compare it with other methods and show that this method is efficient robust and adequate for solving other type of NPDEs. Moreover the Riccati-Bernoulli sub-ODE method gives infinite sequence of solutions. Actually all presented solutions have so important contribution for the explanation of some practical physical problems.

The rest of this work is given as follows: In section 2 we prescribe the $\exp (-\varphi(\xi))$-expansion method, the sine-cosine method and the RiccatiBernoulli sub-ODE method. In Section 3 these methods are applied to solve the Maccari system. In Section 4, some three-dimensional graphs of some solutions is provided. Finally, in Section 5 we give the summary of our contribution.

\section{Analysis of the Methods}

Any nonlinear evolution equation can be presented in following form:

$$
P\left(u, u_{t}, u_{x}, u_{t t}, u_{x x}, \ldots .\right)=0,
$$

where $\mathrm{P}$ is a polynomial in $\mathrm{u}(x, t)$ and its partial derivatives in which the highest order derivatives. The basic steps for this method are

Using the wave transformation for a positive constant $c$,

$u(x, t)=u(\xi), \quad \xi=x-c t$,

Eqn. (1) will be transformed to the following ODE:

$$
D\left(u, u^{\prime}, u^{\prime \prime}, u^{\prime \prime \prime}, \ldots . .\right)=0,
$$

\section{The $\exp (-\varphi(\xi))$-expansion method}

Assume that the solution of eqn. (3) can be written in a polynomial form of $\exp (-\varphi(\xi))$ as follows $[29,30]$ :

$$
u(\xi)=\sum_{m=0}^{n} a_{m}(\exp (-\varphi(\xi)))^{m}, a_{n} \neq 0,
$$

where $\varphi(\xi)$ satisfies the following OD

$$
\varphi^{\prime}(\xi)=\exp (-\varphi(\xi))+\mu \exp (\varphi(\xi))+\lambda .
$$

Eqn. (5) has the following solutions:

1. At $\lambda^{2}-4 \mu>0, \mu \neq 0$,

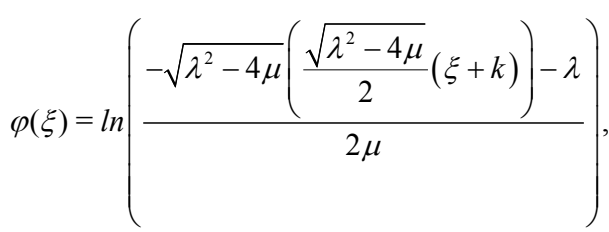

Where $k$ is an arbitrary constant.

*Corresponding author: Abdelrahman MAE, Professor, Department of Mathematics, Faculty of Science, Mansoura University, Egypt, Tel:+02255856568; E-mail: mahmoud.abdelrahman@mans.edu.eg

Received January 25, 2018; Accepted March 13, 2018; Published March 16 2018

Citation: Abdelrahman MAE, Hassan SZ (2018) New Exact Solutions for the Maccari System. J Phys Math 9: 264. doi: 10.4172/2090-0902.1000264

Copyright: @ 2018 Abdelrahman MAE. This is an open-access article distributed under the terms of the Creative Commons Attribution License, which permits unrestricted use, distribution, and reproduction in any medium, provided the original author and source are credited. 
2. At $\lambda^{2}-4 \mu<0, \mu \neq 0$,

$\varphi(\xi)=\ln \left(\frac{\sqrt{4 \mu-\lambda^{2}}\left(\frac{\sqrt{4 \mu-\lambda^{2}}}{2}(\xi+k)\right)-\lambda}{2 \mu}\right)$,

3. At $\lambda^{2}-4 \mu>0, \mu=0, \lambda \neq 0$

$\varphi(\xi)=-\ln \left(\frac{\lambda}{\exp (\lambda(\xi+k))-1}\right)$,

4. At $\lambda^{2}-4 \mu=0, \mu \neq 0, \lambda \neq 0$,

$\varphi(\xi)=\ln \left(-\frac{2(\lambda(\xi+k)+2)}{\lambda^{2}(\xi+k)}\right)$,

5. At $\lambda^{2}-4 \mu=0, \mu=0, \lambda=0$,

$\varphi(\xi)=\ln (\xi+k)$.

Finally, superseding eqn. (4) with eqn. (5) into eqn. (3) and aggregating all terms of the same power

$\exp (m-\varphi(\xi)), \mathrm{m}=0,1,2,3,4 \ldots$ Then equating each term to zero to get algebraic equations which can be solved analytically or by using Mathematica or Maple to obtain the values of $a_{i}$ Hence, we get the solutions (4), which give the exact solutions of eqn. (3).

\section{The sine-cosine method}

The solutions of the reduced ODE eqn. (3) can be expressed in the form $[31,32]$

$$
u(x, t)=\left\{\begin{array}{cc}
\lambda \sin ^{r}(\mu \xi), & |\xi| \leq \frac{\pi}{\mu}, \\
0, & \text { otherwise, }
\end{array}\right.
$$

or in the form

$$
u(x, t)=\left\{\begin{array}{cc}
\lambda \cos ^{r}(\mu \xi), & |\xi| \leq \frac{\pi}{2 \mu}, \\
0, & \text { otherwise, }
\end{array}\right.
$$

where $\lambda, \mu$ and $r^{1} 0$, are parameters, which will be determined. From eqn. (11) we have

$$
\begin{aligned}
& u(\xi)=\lambda \sin ^{r}(\mu \xi), \\
& u^{n}(\xi)=\lambda^{n} \sin ^{n r}(\mu \xi), \\
& \left(u^{n}\right)_{\xi}=n \mu r \lambda^{n} \cos (\mu \xi) \sin ^{n r-1}(\mu \xi), \\
& \left(u^{n}\right)_{\xi \xi}=-n^{2} \mu^{2} r^{2} \lambda^{n} \sin ^{n r}(\mu \xi)+n \mu^{2} \lambda^{n} r(n r-1) \sin ^{n r-2}(\mu \xi),
\end{aligned}
$$

and from (12) we have

$$
\begin{aligned}
& u(\xi)=\lambda \cos ^{r}(\mu \xi), \\
& u^{n}(\xi)=\lambda^{n} \cos ^{n r}(\mu \xi), \\
& \left(u^{n}\right)^{\prime}=-n \mu r \lambda^{n} \sin (\mu \xi) \cos ^{n r-1}(\mu \xi), \\
& \left(u^{n}\right)^{\prime \prime}=-n^{2} \mu^{2} r^{2} \lambda^{n} \cos ^{n r}(\mu \xi)+n \mu^{2} \lambda^{n} r(n r-1) \cos ^{n r-2}(\mu \xi),
\end{aligned}
$$

and so on for other derivatives.

Finally, we substitute the above equations into the reduced equation obtained above in eqn. (3), balance the terms of the cosine functions when (11a) is used, or balance the terms of the sine functions when (12a) is used, and solving the resulting system of algebraic equations by using the computerized symbolic calculations. We next collect all terms with the same power in $\cos ^{k}(\mu \xi)$ or $\sin ^{k}(\mu \xi)$ and set to zero their coefficient to get a system of algebraic equations in the unknowns $\lambda, \mu$ and $r \mathrm{We}$ obtained all possible values of the parameters $\lambda, \mu$ and $r$

\section{Riccati-Bernoulli sub-ODE method}

Due to the Riccati-Bernoulli Sub-ODE technique [16,17,33], we assume that eqn. (3) has a solution given by:

$$
u^{\prime}=a u^{2-n}+b u+c u^{n},
$$

where $a, b, c$ and $n$ are constants determined later. From eqn. (13), we get

$$
\begin{aligned}
u^{\prime \prime} & =a b(3-n) u^{2-n}+a^{2}(2-n) u^{3-2 n}+n c^{2} u^{2 n-1}+b c(n+1) u^{n}+\left(2 a c+b^{2}\right) u, \\
u^{\prime \prime \prime} & =\left(a b(3-n)(2-n) u^{1-n}+a^{2}(2-n)(3-2 n) u^{2-2 n}\right. \\
& \left.+n(2 n-1) c^{2} u^{2 n-2}+b c n(n+1) u^{n-1}+\left(2 a c+b^{2}\right)\right) u^{\prime} .
\end{aligned}
$$

The solitary solutions $u_{i}(\xi)$ of eqn. (13) are given as follow:

1. For $n=1$

$u(\xi)=\mu e^{(a+b+c) \xi}$.

Where $\mu$ is an arbitrary constant.

2. For $n \neq 1 \quad b=0$ and $\mathrm{c}=0$

$$
u(\xi)=(a(n-1)(\xi+\mu))^{\frac{1}{n-1}} \text {. }
$$

3. For $n \neq 1, b \neq 0$ and $c=0$

$u(\xi)=\left(\frac{-a}{b}+\mu e^{b(n-1) \xi}\right)^{\frac{1}{n-1}}$.

4. For $n \neq 1, a \neq 0$ and $b^{2}-4 \mathrm{ac}<0$

$u(\xi)=\left(\frac{-b}{2 a}+\frac{\sqrt{4 a c-b^{2}}}{2 a} \tan \left(\frac{(1-n) \sqrt{4 a c-b^{2}}}{2}(\xi+\mu)\right)\right)^{\frac{1}{1-n}}$

and

$u(\xi)=\left(\frac{-b}{2 a}-\frac{\sqrt{4 a c-b^{2}}}{2 a} \cot \left(\frac{(1-n) \sqrt{4 a c-b^{2}}}{2}(\xi+\mu)\right)\right)^{\frac{1}{1-n}}$.

5. For $n \neq 1, a \neq 0$ and $b^{2}-4 \mathrm{ac}>0$

$u(\xi)=\left(\frac{-b}{2 a}-\frac{\sqrt{b^{2}-4 a c}}{2 a} \operatorname{coth}\left(\frac{(1-n) \sqrt{b^{2}-4 a c}}{2}(\xi+\mu)\right)\right)^{\frac{1}{1-n}}$

and

$u(\xi)=\left(\frac{-b}{2 a}-\frac{\sqrt{b^{2}-4 a c}}{2 a} \tanh \left(\frac{(1-n) \sqrt{b^{2}-4 a c}}{2}(\xi+\mu)\right)\right)^{\frac{1}{1-n}}$

6. For $n \neq 1, a \neq 0$ and $b^{2}-4 \mathrm{ac}=0$

$u(\xi)=\left(\frac{1}{a(n-1)(\xi+\mu)}-\frac{b}{2 a}\right)^{\frac{1}{1-n}}$.

Bäcklund transformation: When $u_{m-1}(\xi)$ and 
$\frac{d u_{m}(\xi)}{d \xi}=\frac{d u_{m}(\xi)}{d u_{m-1}(\xi)} \frac{d u_{m-1}(\xi)}{d \xi}=\frac{d u_{m}(\xi)}{d u_{m-1}(\xi)}\left(a u_{m-1}^{2-n}+b u_{m-1}+c u_{m-1}^{n}\right)$

namely

$$
\frac{d u_{m}(\xi)}{a u_{m}^{2-n}+b u_{m}+c u_{m}^{n}}=\frac{d u_{m-1}(\xi)}{a u_{m-1}^{2-n}+b u_{m-1}+c u_{m-1}^{n}} .
$$

Integrating eqn. (24) once with respect to $\xi$, we get a Bäcklund transformation of eqn. (13) as follows:

$$
u_{m}(\xi)=\left(\frac{-c K_{1}+a K_{2}\left(u_{m-1}(\xi)\right)^{1-n}}{b K_{1}+a K_{2}+a K_{1}\left(u_{m-1}(\xi)\right)^{1-n}}\right)^{\frac{1}{1-n}} .
$$

where $\mathrm{K}_{1}$ and $\mathrm{K}_{2}$ are arbitrary constants. We use eqn. (25) to obtain infinite sequence of solutions of eqn. (13), as well of eqn. (1)

\section{The Maccari System}

Maccari [34] derived a new integrable $(2+1)$ dimensional nonlinear system. This system is obtained by an asymptotically exact reduction method based on Fourier expansion and spatio-temporal rescaling, from the Kadomtsevâ $€$ "Petviashvili equation. The Maccari system is frequently introduced to define the motion of the isolated waves, localized in a very small part of space, in many fields such as hydrodynamics, quantum field theory, nonlinear optics. Indeed, it is plays an important role in plasma physics to describe the behavior of the sonic Langmuir solitons. In this section we are concerned with the Maccari system [35-37], given as follows:

$$
\begin{aligned}
& i \phi_{t}+\phi_{x x}+\phi \psi=0, \\
& \psi_{t}+\psi_{y}+(|\phi|)^{2}=0,
\end{aligned}
$$

Where $\varphi=\varphi(x, y, t)$ and $\psi=\psi(x, y, t)$, represent, respectively, the complex scalar field and the real scalar field. Here $x, y$ are the independent spatial variables and $t>0$ represents the temporal variable. In this section we apply the expansion $\exp (-(\xi))$ method, the sine-cosine method and the Riccati-Bernoulli sub-ODE method, to construct exact solutions for the Maccari system.

\section{Consider the wave transformation}

$$
\phi(x, y, t)=e^{i \varepsilon} u(\xi), \quad \psi(x, y, t)=\psi(\xi), \quad \xi=c_{1} x+c_{2} y+c_{3} t, \quad \varepsilon=k_{1} x+k_{2} y+k_{3} t,
$$

where $c_{1}, c_{2}, c_{3}, k_{1} k_{2}$ and $k_{3}$ are arbitrary constants. Then, eqn. (26) are transformed into the following equations

$$
\begin{aligned}
& i c_{3} u^{\prime}-k_{3} u-k_{1}^{2} u+2 i k_{1} c_{1} u^{\prime}+c_{1}^{2} u^{\prime \prime}+u \psi=0, \\
& \left(c_{2}+c_{3}\right) \psi^{\prime}+c_{1}\left(u^{2}\right)^{\prime}=0,
\end{aligned}
$$

Integrating second equation of eqn. (28) and taking integration constant zero for the sake of simplicity, yields

$$
\psi=\left(\frac{-c_{1}}{c_{2}+c_{3}}\right) u^{2} .
$$

Now by separating real and imaginary parts in the first equation of (28), we have

$$
\begin{aligned}
& i\left(c_{3}+2 k_{1} c_{1}\right) u^{\prime}=-0 . \\
& -\left(k_{3}+k_{1}^{2}\right) u+c_{1}^{2} u^{\prime \prime}+u \psi=0 .
\end{aligned}
$$

Substituting eqn. (29) into (31), we get the following NODE:

$$
l_{1} u^{\prime \prime}+l_{2} u^{3}+l_{3} u=0
$$

where $l_{1}=c_{1}^{2}, l_{2}=\left(\frac{-c_{1}}{c_{2}+c_{3}}\right), l_{3}=-\left(k_{1}^{2}+k_{3}\right)$.

\section{On solving eqn. (26) using the $\exp (-\varphi(\xi))$-expansion method}

Balancing the highest order derivative $u^{\prime \prime}$ and non-linear term $u^{3}$ in eqn. (32), we obtain $n=1$. Consequently, eqn. (32) has the following solution

$$
U=A_{0}+A_{1} \exp (-\varphi),
$$

where $\mathrm{A}_{0}$ and $\mathrm{A}_{1}$ are constants to be determined, such that $\mathrm{A}_{1} \neq 0$ It is easy to see that

$$
\begin{aligned}
& U^{\prime \prime}=A_{1}\left(2 \exp (-3 \varphi)+3 \lambda \exp (-2 \varphi)+\left(2 \mu+\lambda^{2}\right) \exp (-\varphi)+\lambda \mu\right), \\
& U^{3}=A_{1}^{3} \exp (-3 \varphi)+3 A_{0} A_{1}^{2} \exp (-2 \varphi)+3 A_{0}^{2} A_{1} \exp (-\varphi)+A_{0}^{3} .
\end{aligned}
$$

Superseding $u, u^{\prime \prime}, u^{3}$ into eqn. (32) and then equating the coefficients of $\exp \varphi$ ) to zero, we obtain

$$
\begin{aligned}
& l_{1} A_{1} \lambda \mu+l_{2} A_{0}^{3}+l_{3} A_{0}=0, \\
& A_{1} l_{1}\left(\lambda^{2}+2 \mu\right)+3 l_{2} A_{0}^{2} A_{1}+l_{3} A_{1}=0, \\
& 3 l_{1} A_{1} \lambda+3 l_{2} A_{0} A_{1}^{2}=0, \\
& 2 l_{1} A_{1}+l_{2} A_{1}^{3}=0 .
\end{aligned}
$$

Solving eqns. (36)-(39), we get

$$
l_{1}=\frac{2 l_{3}}{\lambda^{2}-4 \mu}, A_{0}= \pm \lambda \sqrt{\frac{l_{3}}{l_{2}\left(4 \mu-\lambda^{2}\right)}}, A_{1}= \pm 2 \sqrt{\frac{l_{3}}{l_{2}\left(4 \mu-\lambda^{2}\right)}} .
$$

Actually we will consider only case from the above cases, the other cases follow in the same way. Now substituting the values of $A_{0}, A_{1}$ into eqn. (33) yields

$$
U(\xi)= \pm \sqrt{\frac{l_{3}}{l_{2}\left(4 \mu-\lambda^{2}\right)}}(\lambda+2 \exp (-\varphi(\xi))) .
$$

Now substituting eqns. (6)-(7) into eqn. (40) respectively, we have the following solutions:

Case 1. At $\lambda^{2}-4 \mu>0, \mu \neq 0$,

$$
u_{1,2}(x, y, t)= \pm \sqrt{\frac{l_{3}}{l_{2}\left(4 \mu-\lambda^{2}\right)}}\left(\lambda-\frac{4 \mu}{\sqrt{\lambda^{2}-4 \mu} \tanh \left(\frac{\sqrt{\lambda^{2}-4 \mu}}{2}\left(c_{1} x+c_{2} y+c_{3} t+k\right)\right)+\lambda}\right),
$$

where $l_{2}, l_{3}, c_{1}, c_{2}, c_{3}, \lambda, \mu$ and $k$ are arbitrary constants. Using eqns. (41), (27) and (29) the solutions of eqn. (26) take the forms:

$$
\phi_{1,2}(x, y, t)= \pm e^{i\left(k_{1}+k+k_{2} y k_{3}\right)} \sqrt{\frac{l_{3}}{l_{2}\left(4 \mu-\lambda^{2}\right)}}\left(\lambda-\frac{4 \mu}{\sqrt{\lambda^{2}-4 \mu} \tanh \left(\frac{\sqrt{\lambda^{2}-4 \mu}}{2}\left(c_{1} x+c_{2} y+c_{3} t+k\right)\right)+\lambda}\right)(42)
$$

and

$$
\psi_{1,2}(x, y, t)=\left(\frac{-c_{1}}{c_{2}+c_{3}}\right)\left(\sqrt{\frac{l_{3}}{l_{2}\left(4 \mu-\lambda^{2}\right)}}\left(\lambda-\frac{4 \mu}{\sqrt{\lambda^{2}-4 \mu} \tanh \left(\frac{\sqrt{\lambda^{2}-4 \mu}}{2}\left(c_{1} x+c_{2} y+c_{3} t+k\right)\right)+\lambda}\right)\right)^{2}
$$

Case 2. At $\lambda^{2}-4 \mu<0, \mu \neq 0$,

$$
u_{3,4}(x, y, t)= \pm \sqrt{\frac{l_{3}}{l_{2}\left(4 \mu-\lambda^{2}\right)}}\left(\lambda+\frac{4 \mu}{\sqrt{4 \mu-\lambda^{2}} \tanh \left(\frac{\sqrt{4 \mu-\lambda^{2}}}{2}\left(c_{1} x+c_{2} y+c_{3} t+k\right)\right)-\lambda}\right) .
$$


where $l_{2}, l_{3}, c_{1}, c_{2}, c_{3}, \lambda, \mu$ and $k$ are arbitrary constants.

Using eqns. (44), (27) and (29) the solutions of eqn. (26) take the forms:

$$
\phi_{3,4}(x, y, t)= \pm e^{\left.i l_{1}+x+k_{2} y+k_{3}\right)} \sqrt{\frac{l_{3}}{l_{2}\left(4 \mu-\lambda^{2}\right)}}\left(\lambda+\frac{4 \mu}{\sqrt{4 \mu-\lambda^{2}} \tanh \left(\frac{\sqrt{4 \mu-\lambda^{2}}}{2}\left(c_{1} x+c_{2} y+c_{3} t+k\right)\right)-\lambda}\right)
$$

and

$$
\psi_{3,4}(x, y, t)=\left(\frac{-c_{1}}{c_{2}+c_{3}}\right)\left(\sqrt{\frac{l_{3}}{l_{2}\left(4 \mu-\lambda^{2}\right)}}\left(\lambda+\frac{4 \mu}{\sqrt{4 \mu-\lambda^{2}} \tanh \left(\frac{\sqrt{4 \mu-\lambda^{2}}}{2}\left(c_{1} x+c_{2} y+c_{3} t+k\right)\right)-\lambda}\right)\right)^{2}
$$

Case 3. At $\lambda^{2}-4 \mu>0, \mu=0, \lambda \neq 0$

$$
u_{5,6}(x, y, t)= \pm \sqrt{\frac{l_{3}}{-l_{2} \lambda^{2}}}\left(\lambda+\frac{2 \lambda}{\exp \left(\lambda\left(c_{1} x+c_{2} y+c_{3} t+k\right)\right)-1}\right) .
$$

Using eqns. (47), (27) and (29) the solutions of eqn. (26) take the forms:

$$
\phi_{5,6}(x, y, t)= \pm e^{i\left(k_{1} x+k_{2} y+k_{3} t\right)} \sqrt{\frac{l_{3}}{-l_{2} \lambda^{2}}}\left(\lambda+\frac{2 \lambda}{\exp \left(\lambda\left(c_{1} x+c_{2} y+c_{3} t+k\right)\right)-1}\right)
$$

and

$$
\psi_{3,4}(x, y, t)=\left(\frac{-c_{1}}{c_{2}+c_{3}}\right)\left(\sqrt{\frac{l_{3}}{-l_{2} \lambda^{2}}}\left(\lambda+\frac{2 \lambda}{\exp \left(\lambda\left(c_{1} x+c_{2} y+c_{3} t+k\right)\right)-1}\right)\right)^{2},
$$

where $l_{2}, l_{3}, c_{1}, c_{2}, c_{3}, \lambda, \mu$ and $k$ are arbitrary constants.

\section{On solving eqn. (26) using the sine-cosine method}

Now we apply the sine-cosine method for solving eqn. (32). Subtitling (31) into (32), gives

$l_{1}\left(-\mu^{2} r^{2} \lambda \sin ^{r}(\mu \xi)+\mu^{2} \lambda r(r-1) \sin ^{r-2}(\mu \xi)\right)+l_{2} \lambda^{3} \sin ^{3 r}(\mu \xi)+l_{3} \lambda \sin ^{r}(\mu \xi)=0$.

Equating the exponents and the coefficient of the sine functions gives the following system of algebraic equations:

$$
\begin{aligned}
& r-1 \neq 0, \quad r-2=3 r, \\
& l_{1} \mu^{2} \lambda r(r-1)+l_{2} \lambda^{3}=0, \\
& -l_{1} \mu^{2} r^{2} \lambda+l_{3} \lambda=0 .
\end{aligned}
$$

Solving this system gives

$$
r=-1, \quad \lambda= \pm i \sqrt{\frac{2 l_{3}}{l_{2}}}, \quad \mu= \pm \sqrt{\frac{l_{3}}{l_{1}}},
$$

for $\frac{l_{3}}{l_{1}}>0$ and $\frac{l_{3}}{l_{2}}>0$ with $l_{1}, l_{2}{ }^{1} 0$. The result (52) can be easily obtained if we also use the cosine method (12). Consequently, we get the :following periodic solutions

$$
\begin{aligned}
& \tilde{u}_{1}(x, y, t)= \pm \sqrt{\frac{2 l_{3}}{l_{2}}} \sec \left(\sqrt{\frac{l_{3}}{l_{1}}}\left(c_{1} x+c_{2} y+c_{3} t\right)\right),\left|\sqrt{\frac{l_{3}}{l_{1}}}\left(c_{1} x+c_{2} y+c_{3} t\right)\right|<\frac{\pi}{2} \\
& \text { and } \\
& \tilde{u}_{2}(x, y, t)= \pm \sqrt{\frac{2 l_{3}}{l_{2}}} \csc \left(\sqrt{\frac{l_{3}}{l_{1}}}\left(c_{1} x+c_{2} y+c_{3} t\right)\right), 0<\sqrt{\frac{l_{3}}{l_{1}}}\left(c_{1} x+c_{2} y+c_{3} t\right)<\pi .
\end{aligned}
$$

Using eqns. (53), (54), (27) and (29) the solutions of eqn. (26) take the forms:

$$
\begin{aligned}
& \tilde{\phi}_{1}(x, y, t)= \pm e^{i\left(l_{1} x+k_{2} y+k_{3} t\right)} \sqrt{\frac{2 l_{3}}{l_{2}}} \sec \left(\sqrt{\frac{l_{3}}{l_{1}}}\left(c_{1} x+c_{2} y+c_{3} t\right)\right),\left|\sqrt{\frac{l_{3}}{l_{1}}}\left(c_{1} x+c_{2} y+c_{3} t\right)\right|<\frac{\pi}{2}, \\
& \tilde{\phi}_{2}(x, y, t)= \pm e^{i\left(k_{1} x+k_{2} y+k_{3} t\right)} \sqrt{\frac{2 l_{3}}{l_{2}}} \operatorname{ssc}\left(\sqrt{\frac{l_{3}}{l_{1}}}\left(c_{1} x+c_{2} y+c_{3} t\right)\right), 0<\sqrt{\frac{l_{3}}{l_{1}}}\left(c_{1} x+c_{2} y+c_{3} t\right)<\pi, \\
& \tilde{\psi}_{1}(x, y, t)=\left(\frac{-c_{1}}{c_{2}+c_{3}}\right)\left(\sqrt{\frac{2 l_{3}}{l_{2}}} \sec \left(\sqrt{\frac{l_{3}}{l_{1}}}\left(c_{1} x+c_{2} y+c_{3} t\right)\right)\right)^{2},\left|\sqrt{\frac{l_{3}}{l_{1}}}\left(c_{1} x+c_{2} y+c_{3} t\right)\right|<\frac{\pi}{2}
\end{aligned}
$$

and

$$
\tilde{\psi}_{2}(x, y, t)=\left(\frac{-c_{1}}{c_{2}+c_{3}}\right)\left(\sqrt{\frac{2 l_{3}}{l_{2}}} \csc \left(\sqrt{\frac{l_{3}}{l_{1}}}\left(c_{1} x+c_{2} y+c_{3} t\right)\right)\right)^{2}, 0<\sqrt{\frac{l_{3}}{l_{1}}}\left(c_{1} x+c_{2} y+c_{3} t\right)<\pi .
$$

However, for $\frac{l_{3}}{l_{2}}<0$ and $l_{2}{ }^{1} 0$, we obtain the soliton and complex
lutions

$$
\tilde{u}_{3}(x, y, t)= \pm \sqrt{\frac{2 l_{3}}{l_{2}}} \operatorname{sech}\left(\sqrt{-\frac{l_{3}}{l_{1}}}\left(c_{1} x+c_{2} y+c_{3} t\right)\right)
$$

and

$$
\tilde{u}_{4}(x, y, t)= \pm \sqrt{\frac{2 l_{3}}{l_{2}}} \operatorname{csch}\left(\sqrt{\frac{l_{3}}{l_{1}}}\left(c_{1} x+c_{2} y+c_{3} t\right)\right) .
$$

Using eqns. (59), (60), (27) and (29), we get the solutions of eqn. (26) in the same way as in eqns. (55)-(58).

\section{On solving eqn. (26) using the Riccati-Bernoulli Sub-ODE method}

Now we apply the Riccati-Bernoulli Sub-ODE method for solving eqn. (32). Substituting eqn. (14) into eqn. (32), we obtain

$l_{1}\left(a b(3-n) u^{2-n}+a^{2}(2-n) u^{3-2 n}+n c^{2} u^{2 n-1}+b c(n+1) u^{n}+\left(2 a c+b^{2}\right) u\right)+l_{2} u^{3}+l_{3} u=0$

Setting $n=0$, eqn. (61) is reduced to

$l_{1}\left(3 a b u^{2}+2 a^{2} u^{3}+b c+\left(2 a c+b^{2}\right) u\right)+l_{2} u^{3}+l_{3} u=0$.

Putting each coefficient o $u^{i}(i=0,1,2,3)$ to zero, we get

$l_{1} b c=0$,

$l_{1}\left(2 a c+b^{2}\right)+l_{3}=0$,

$3 l_{1} a b=0$,

$2 l_{1} a^{2}+l_{2}=0$

Solving eqns. (63)-(66), we get

$b=0$

$c=\mp \frac{l_{3}}{\sqrt{-2 l_{1} l_{2}}}$,

$a= \pm \sqrt{\frac{-l_{2}}{2 l_{1}}}$.

Hence, we give the cases of solutions for the eqns. (32) and (26), respectively

1. When $b=0$ and $c=0$ the solution of eqn. (32) is

$\hat{u}_{1}(x, y, t)=\left(-a\left(c_{1} x+c_{2} y+c_{3} t+\mu\right)\right)^{-1}$.

Using eqns. (70), (27) and (29) the solutions of eqn. (26) take the forms:

$$
\hat{\phi}_{1}(x, y, t)=e^{i\left(k_{1} x+k_{2} y+k_{3} t\right)}\left(-a\left(c_{1} x+c_{2} y+c_{3} t+\mu\right)\right)^{-1},
$$




$$
\hat{\psi}_{1}(x, t)=\left(\frac{-c_{1}}{c_{2}+c_{3}}\right)\left(\left(-a\left(c_{1} x+c_{2} y+c_{3} t+\mu\right)\right)^{-1}\right)^{2},
$$

where $k_{1}, k_{2}, k_{3}, c_{1}, c_{2}, c_{3}, \mu$ are arbitrary constants.

2. When $\frac{l_{3}}{l_{1}}<0$, substituting eqns. (67)-(69), (27) and (29) into eqns. (19) and (20), we obtain the exact solutions of eqn. (26) as follows

$$
\hat{u}_{2,3}(x, y, t)= \pm \sqrt{\frac{l_{3}}{l_{2}}} \tan \left(\sqrt{\frac{-l_{3}}{l_{1}}}\left(c_{1} x+c_{2} y+c_{3} t+\mu\right)\right)
$$

and

$$
\hat{u}_{4,5}(x, y, t)= \pm \sqrt{\frac{l_{3}}{l_{2}}} \cot \left(\sqrt{\frac{-l_{3}}{l_{1}}}\left(c_{1} x+c_{2} y+c_{3} t+\mu\right)\right) .
$$

Using eqns. (73), (74), (27) and (29) the solutions of eqn. (26) take the forms:

$$
\hat{\phi}_{2,3}(x, y, t)= \pm \sqrt{\frac{l_{3}}{l_{2}}} e^{i((x+k t)} \tan \left(\sqrt{\frac{-l_{3}}{l_{1}}}\left(c_{1} x+c_{2} y+c_{3} t+\mu\right)\right)
$$

and

$$
\begin{aligned}
& \hat{\phi}_{4,5}(x, y, t)= \pm \sqrt{\frac{l_{3}}{l_{2}}} e^{i\left(k_{1} x+k_{2} y+k_{3} t\right)} \cot \left(\sqrt{\frac{-l_{3}}{l_{1}}}\left(c_{1} x+c_{2} y+c_{3} t+\mu\right)\right), \\
& \hat{\psi}_{2,3}(x, y, t)=\left(\frac{-c_{1}}{c_{2}+c_{3}}\right)\left(\sqrt{\frac{l_{3}}{l_{2}}} \tan \left(\sqrt{\frac{-l_{3}}{l_{1}}}\left(c_{1} x+c_{2} y+c_{3} t+\mu\right)\right)\right)^{2}, \\
& \hat{\psi}_{4,5}(x, y, t)=\left(\frac{-c_{1}}{c_{2}+c_{3}}\right)\left(\sqrt{\frac{l_{3}}{l_{2}}} \cot \left(\sqrt{\frac{-l_{3}}{l_{1}}}\left(c_{1} x+c_{2} y+c_{3} t+\mu\right)\right)\right)^{2},
\end{aligned}
$$

Where $k_{1}, k_{2}, k_{3}, c_{1}, c_{2}, c_{3}, l_{\mathrm{i}}, l_{2}, l_{3}, \mu$ are arbitrary constants.

3. When $\frac{l_{3}}{l_{1}}>0$, substituting eqns. (67)-(69) and (27) into eqns. (21) and (22), we get exact solutions of eqn. (26),

$$
\hat{u}_{6,7}(x, y, t)= \pm \sqrt{\frac{-l_{3}}{l_{2}}} \tanh \left(\sqrt{\frac{l_{3}}{l_{1}}}\left(c_{1} x+c_{2} y+c_{3} t+\mu\right)\right)
$$

and

$$
\hat{u}_{8,9}(x, y, t)= \pm \sqrt{\frac{-l_{3}}{l_{2}}} \operatorname{coth}\left(\sqrt{\frac{l_{3}}{l_{1}}}\left(c_{1} x+c_{2} y+c_{3} t+\mu\right)\right) \text {. }
$$

Using eqns. (79), (80), (27) and (29) the solutions of eqn. (26) take the forms:

$$
\hat{\phi}_{6,7}(x, y, t)= \pm \sqrt{\frac{-l_{3}}{l_{2}}} e^{i\left(k_{1} x+k_{2} y+k_{3} t\right)} \tanh \left(\sqrt{\frac{l_{3}}{l_{1}}}\left(c_{1} x+c_{2} y+c_{3} t+\mu\right)\right)
$$

and

$$
\begin{aligned}
& \hat{\phi}_{8,9}(x, y, t)= \pm \sqrt{\frac{-l_{3}}{l_{2}}} e^{i\left(k_{1} x+k_{2} y+k_{3} t\right)} \tanh \left(\sqrt{\frac{l_{3}}{l_{1}}}\left(c_{1} x+c_{2} y+c_{3} t+\mu\right)\right), \\
& \hat{\psi}_{6,7}(x, y, t)=\left(\frac{-c_{1}}{c_{2}+c_{3}}\right)\left(\sqrt{\frac{-l_{3}}{l_{2}}} \tanh \left(\sqrt{\frac{l_{3}}{l_{1}}}\left(c_{1} x+c_{2} y+c_{3} t+\mu\right)\right)\right)^{2}, \\
& \hat{\psi}_{8,9}(x, y, t)=\left(\frac{-c_{1}}{c_{2}+c_{3}}\right)\left(\sqrt{\frac{-l_{3}}{l_{2}}} \operatorname{coth}\left(\sqrt{\frac{l_{3}}{l_{1}}}\left(c_{1} x+c_{2} y+c_{3} t+\mu\right)\right)\right)^{2},
\end{aligned}
$$

Where $k_{1}, k_{2}, k_{3}, c_{1}, c_{2}, c_{3} l_{\mathrm{i}}, l_{2}, l_{3}, \mu$ are arbitrary constants.

\section{Remark 3.1}

Applying eqn. (25) to $u_{i}(x, y, t) i=1,2, \ldots, 9$, we obtain an infinite sequence of solutions of eqn. (32). Consequently, we get an infinite sequence of solutions of eqn. (26). For illustration, by applying eqn. (25) to $u_{i}(x, y, t) i=1,2, \ldots, 9$, once, we have new solutions of eqn. (32)

$$
\begin{aligned}
& u_{1}^{\star}(x, y, t)=\frac{B_{3}}{-a B_{3}\left(c_{1} x+c_{2} y+c_{3} t+\mu\right) \pm 1}, \\
& u_{2,3}^{\star}(x, y, t)=\frac{ \pm \frac{l_{3}}{\sqrt{-2 l_{1} l_{2}}} \pm B_{3} \sqrt{\frac{l_{3}}{l_{2}}} \tan \left(\sqrt{\frac{-l_{3}}{l_{1}}}\left(c_{1} x+c_{2} y+c_{3} t+\mu\right)\right)}{B_{3} \pm \sqrt{\frac{l_{3}}{l_{2}}} \tan \left(\sqrt{\frac{-l_{3}}{l_{1}}}\left(c_{1} x+c_{2} y+c_{3} t+\mu\right)\right)}, \\
& u_{4,5}^{\star}(x, y, t)=\frac{ \pm \frac{l_{3}}{\sqrt{-2 l_{1} l_{2}}} \pm B_{3} \sqrt{\frac{l_{3}}{l_{2}}} \cot \left(\sqrt{\frac{-l_{3}}{l_{1}}}\left(c_{1} x+c_{2} y+c_{3} t+\mu\right)\right)}{B_{3} \pm \sqrt{\frac{l_{3}}{l_{2}}} \cot \left(\sqrt{\frac{-l_{3}}{l_{1}}}\left(c_{1} x+c_{2} y+c_{3} t+\mu\right)\right)}, \\
& u_{6,7}^{\star}(x, y, t)=\frac{ \pm \frac{l_{3}}{\sqrt{-2 l_{1} l_{2}}} \pm B_{3} \sqrt{\frac{-l_{3}}{l_{2}}} \tanh \left(\sqrt{\frac{l_{3}}{l_{1}}}\left(c_{1} x+c_{2} y+c_{3} t+\mu\right)\right)}{B_{3} \pm \sqrt{\frac{-l_{3}}{l_{2}}} \tanh \left(\sqrt{\frac{l_{3}}{l_{1}}}\left(c_{1} x+c_{2} y+c_{3} t+\mu\right)\right)}, \\
& u_{8,9}^{\star}(x, y, t)=\frac{ \pm \frac{l_{3}}{\sqrt{-2 l_{1} l_{2}}} \pm B_{3} \sqrt{\frac{-l_{3}}{l_{2}}} \operatorname{coth}\left(\sqrt{\frac{l_{3}}{l_{1}}}\left(c_{1} x+c_{2} y+c_{3} t+\mu\right)\right)}{B_{3} \pm \sqrt{\frac{-l_{3}}{l_{2}}} \operatorname{coth}\left(\sqrt{\frac{l_{3}}{l_{1}}}\left(c_{1} x+c_{2} y+c_{3} t+\mu\right)\right)},
\end{aligned}
$$

where $B_{3}, c_{1}, c_{2}, c_{3}, l_{1}, l_{2}, l_{3}, \mu$ are arbitrary constants.

\section{Remark 3.2}

1. Comparing our results concerning eqn. (26) with the results [3537], one can see that our results are new and most extensive. Moreover it can be seen that by choosing suitable values for the parameters similar solutions can be verified

2. One very important characteristic, that the Riccati-Bernoulli Sub-ODE method admits infinite sequence of solutions of equation, which has never given for any another methods.

3. Consequently, the method is efficacious robust and adequate for solving such types PDEs.

\section{Graphs for the Solutions}

In this section $3 \mathrm{D}$ graphics of some solutions have been plotted, namely Figures 1-7.

\section{Conclusion}

In this work we have introduced the $\exp (-\varphi(\xi))$-expansion, the sinecosine methods and the Riccati-Bernoulli sub-ODE method in order to obtain the exact travelling wave solutions for the Maccari system. Many new and more general traveling wave solutions are obtained during the analytical treatment. The calculations demonstrate that this method has a very important role to obtain analytical solutions in a unified and more general form. It is shown that these three methods provide a very effective and powerful mathematical tool for solving nonlinear evolution equations in mathematical physics, such as the schrodinger 


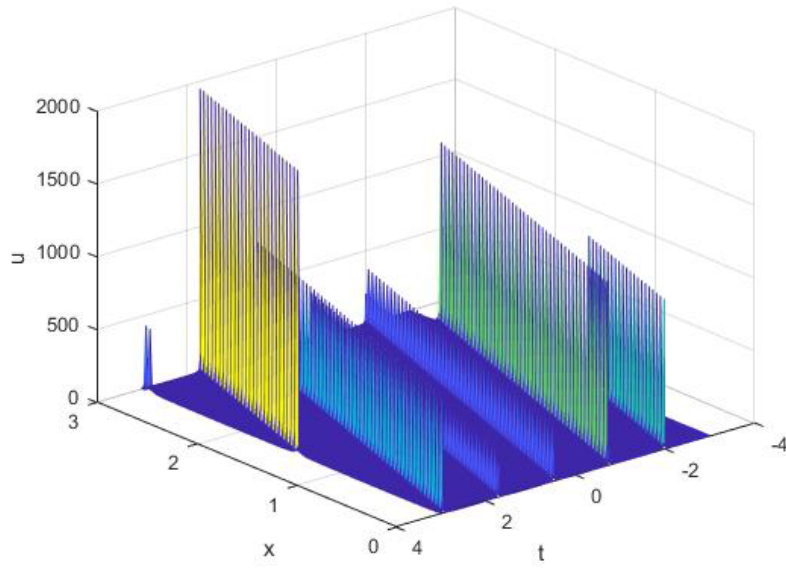

Figure 1: The solution $u=u_{1}$ in eqn. (41) with $l_{2}=1.5, l_{3}=2, m u=2.2, \lambda=3.5$, $l_{1}=1.1594, c_{1}=1, c_{3}=1.6, k=4$ and $-2 \leq x \leq 2,0 \leq t \leq 2$.

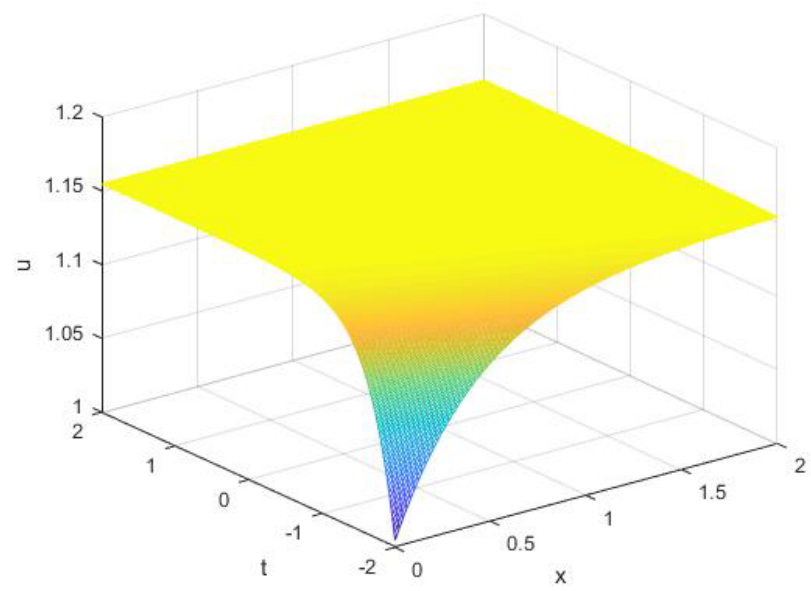

Figure 2: The stochastic solution $u=u_{3}$ in eqn. (44) with $l_{2}=1.5, l_{3}=2, m u=3$, $\lambda=2, l_{1}=-0.5, c_{1}=1.2, c_{3}=1.8, k=4$ and $-3 \leq x \leq 3,0 \leq t \leq 3$.

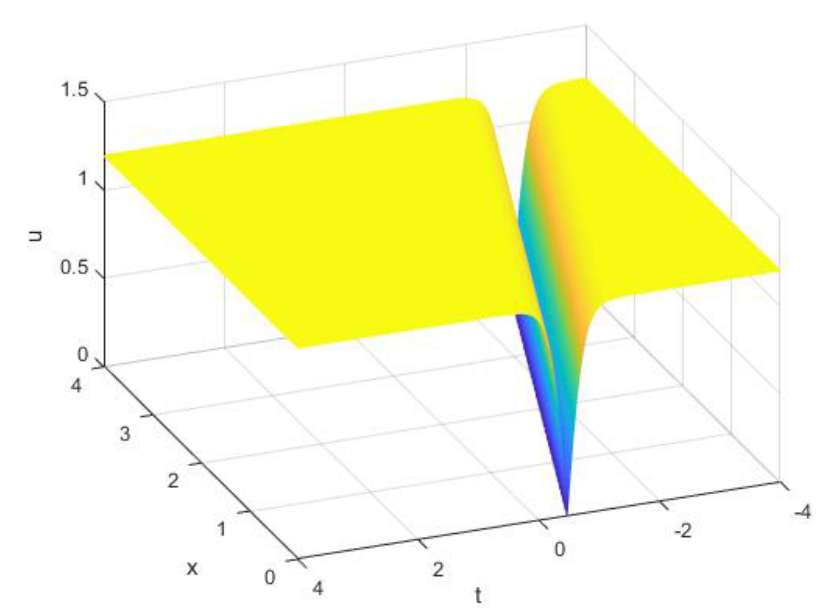

Figure 3: The solution $u=u_{9}$ in eqn. (47) with $l_{2}=2.5, l_{3}=2.4, \quad m u=1.6, \lambda=4$, $l_{1}=0.5, c_{1}=1.2, c_{3}=1.7, k=2$ and $-4 \leq x \leq 4,0 \leq t \leq 4$.

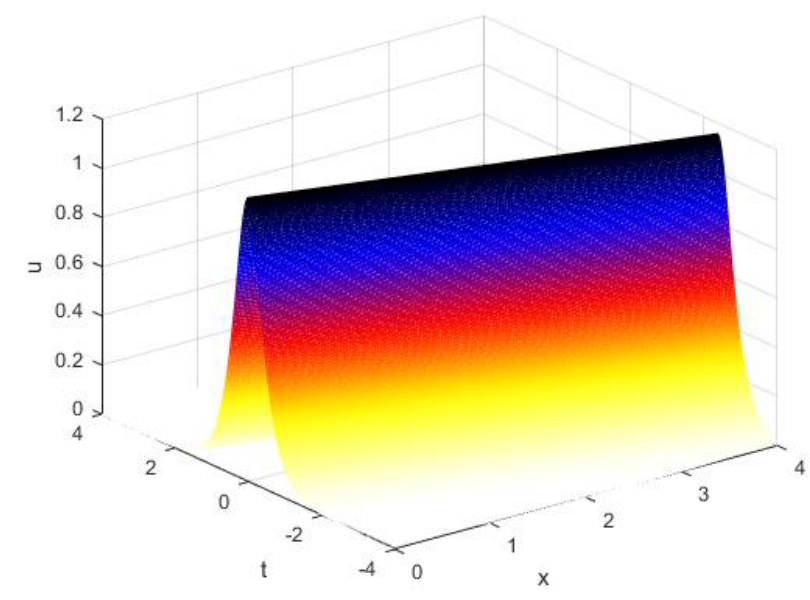

Figure 4: The solution $u=\tilde{u}_{1}$ in eqn. (53) with $l_{1}=1.1, l_{3}=2, c_{1}=1.3, c_{3}=2.2$, and $-4 \leq x \leq 4,0 \leq t \leq 4$.

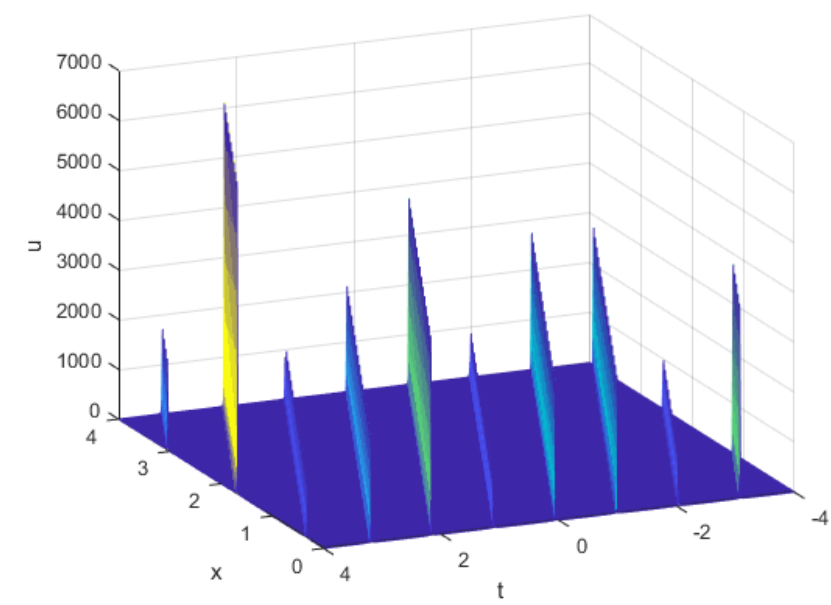

Figure 5: The solution $u=\tilde{u}_{1}$ in eqn. (59) with $l_{1}=1.1, l_{2}=3, l_{3}=-2, c_{1}=1.3$, $c_{3}=2.2$, and $-4 \leq x \leq 4,0 \leq t \leq 4$.

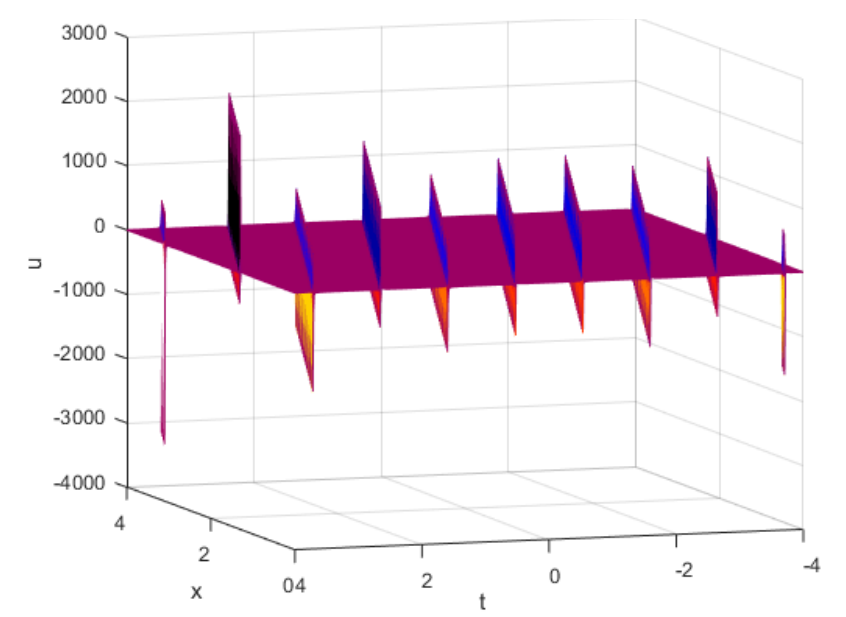

Figure 6: The solution $u=\hat{u}_{2}$ in eqn. (53) with $l_{1}=1.5, l_{2}=1.6, l_{3}=-2, \mu=1.2$, $c_{1}=1.5, c_{3}=2.6$, and $-4 \leq x \leq 4,0 \leq t \leq 4$. 


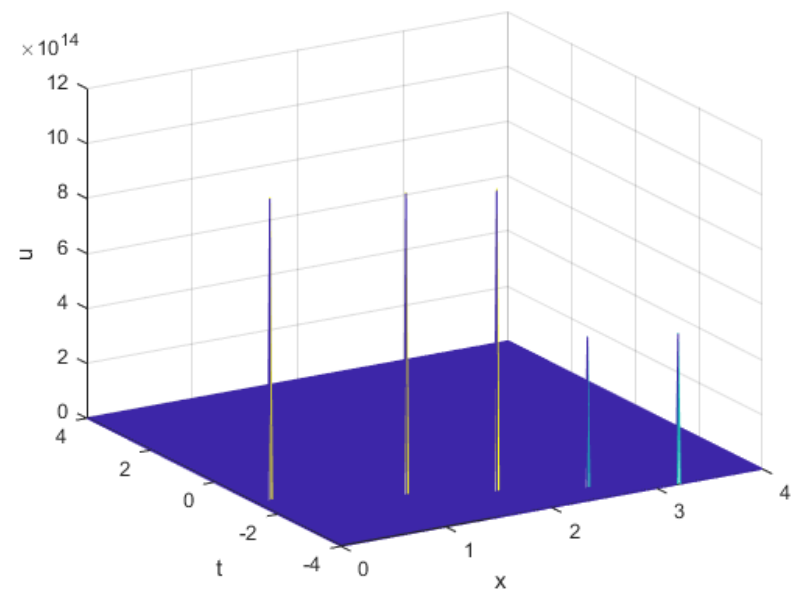

Figure 7: The solution $u=\hat{u}_{6}$ in eqn. (59) with $l_{1}=1.4, l_{2}=3.1, l_{3}=3, \mu=1.2$, $c_{1}=2.5, c_{3}=2.6$, and $-4 \leq x \leq 4,0 \leq t \leq 4$.

equation, the Phi- 4 equation, the 2D Ginzburg-Landau equation, the generalized-Zakharov system, etc.

\section{References}

1. Abdelrahman MAE, Kunik M (2015) The Ultra-Relativistic Euler Equations. Math Meth Appl Sci 38: 247-1264.

2. Abdelrahman MAE (2017) Global Solutions for the Ultra-Relativistic Euler Equations. Nonlinear Analysis, 155: 140-162.

3. Abdelrahman MAE (2017) On the Shallow Water Equations. Z Naturforsch 72: 873-879.

4. Razborova P, Ahmed B, Biswas A (2014) Solitons, shock waves and conservation laws of Rosenau-KdV-RLW equation with power law nonlinearity. Appl Math Inf Sci 8: 485-491.

5. Biswas A, Mirzazadeh M (2014) Dark optical solitons with power law nonlinearity using $G^{\prime} / G$-expansion. Optik 125: 4603-4608.

6. Younis M, Ali S, Mahmood SA (2015) Solitons for compound KdV Burgers equation with variable coefficients and power law nonlinearity. Nonlinear Dyn 81: 1191-1196.

7. Bhrawy AH (2014) An efficient Jacobi pseudospectral approximation for nonlinear complex generalized Zakharov system. Appl Math Comput 247: 30-46.

8. Fan E, Zhang H (1998) A note on the homogeneous balance method. Phys Lett A 246: 403-406.

9. Wang ML (1996) Exact solutions for a compound KdV-Burgers equation. Phys Lett A 213: 279-287.

10. Abdou MA (2007) The extended F-expansion method and its application for a class of nonlinear evolution equations. Chaos Solitons Fractals 31: 95-104.

11. Ren YJ, Zhang HQ (2006) A generalized F-expansion method to find abundant families of Jacobi elliptic function solutions of the (2+1)-dimensional NizhnikNovikov-Veselov equation. Chaos Solitons Fractals 27: 959-979.

12. Zhang JL, Wang ML, Wang YM, Fang ZD (2006) The improved F-expansion method and its applications. Phys Lett A 350: 103-109.

13. Malfliet W (1992) Solitary wave solutions of nonlinear wave equation. Am J Phys 60: 650-654.

14. Malfliet W, Hereman W (1996) The tanh method: Exact solutions of nonlinear evolution and wave equations. Phys Scr 54: 563-568.
15. Wazwaz AM (2004) The tanh method for travelling wave solutions of nonlinear equations. Appl Math Comput 154: 714-723.

16. Abdelrahman MAE, Sohaly MA (2017) Solitary Waves for the Modified Korteweg-De Vries Equation in Deterministic Case and Random Case. J Phys Math 8: 214

17. Abdelrahman MAE, Sohaly MA (2017) Solitary waves for the nonlinear Schrodinger problem with the probability distribution function in stochastic input case. Eur Phys J Plus 132: 339.

18. Dai CQ, Zhang JF (2006) Jacobian elliptic function method for nonlinea differential difference equations. Chaos Solutions Fractals 27: 1042-1049.

19. Fan E, Zhang J (2002) Applications of the Jacobi elliptic function method to special-type nonlinear equations. Phys Lett A 305: 383-392.

20. Wazwaz AM (2005) Exact solutions to the double sinh-Gordon equation by the tanh method and a variable separated ODE. Method. Comput Math Appl 50 1685-1696.

21. Wazwaz AM (2004) A sine-cosine method for handling nonlinear wave equations. Math Comput Modelling 40: 499-508.

22. Yan C (1996) A simple transformation for nonlinear waves. Phys Lett A 224 77-84.

23. EL-Wakil SA, Abdou MA (2007) New exact travelling wave solutions using modified extented tanh-function method. Chaos Solitons Fractals 31: 840-852.

24. Fan $E$ (2000) Extended tanh-function method and its applications to nonlinear equations. Phys Lett A 277: 212-218.

25. Wazwaz AM (2007) The extended tanh method for abundant solitary wave solutions of nonlinear wave equations. Appl Math Comput 187: 1131-1142.

26. Wang ML, Zhang JL Li XZ (2008) The $\left(\frac{G^{\prime}}{G}\right)$ - expansion method and travelling wave solutions of nonlinear evolutions equations in mathematical physics. Phys Lett A 372: 417-423.

27. Zhang S, Tong JL, Wang W (2008) A generalized $\left(\frac{G^{\prime}}{G}\right)$ - expansion method for the mKdv equation with variable coefficients. Phys Lett A 37: 2254-2257.

28. Abdelrahman MAE, Zahran EHM, Khater MMA (2015) The $\operatorname{Exp}(-\phi(\xi))$ -expansion method and its application for solving nonlinear evolution equations. Int J Mod Nonlinear Teory and Application 4: 37-47.

29. He JH, Wu XU (2006) Exp-function method for nonlinear wave equations Chaos Solitons Fractals 30: 700-708.

30. Aminikhad H, Moosaei H, Hajipour M (2009) Exact solutions for nonlinear partial differential equations via Exp-function method. Numer Methods Partia Differ equations 26: 1427-1433.

31. Wazwaz AM (2004) The sine-cosine method for obtaining solutions with compact and noncompact structures. Appl Math Comput 159: 559-576.

32. Tascan F, Bekir A (2009) Analytic solutions of the (2+1)-dimensional nonlinear evolution equations using the sine-cosine method. Appl Math Comput 215 3134-3139.

33. Yang XF, Deng ZC, Wei Y (2015) A Riccati-Bernoulli sub-ODE method for nonlinear partial differential equations and its application. Adv Diff Equa 1: 117-133.

34. Maccari A (1996) The Kadomtsev-Petviashvili equation as a source of integrable model equations. J Math Phys 37: 6207.

35. Cheemaa N, Younis M (2016) New and more exact traveling wave solutions to integrable (2+1)-dimensional Maccari system. Nonlinear Dynamics 83: 13951401.

36. Demiray ST, Pandir Y, Bulut H (2015) New solitary wave solutions of Maccar system. Ocean Eng 103: 153-159.

37. Zhang S (2007) Exp-function method for solving Maccari's system. Phys Let A 371: 65-71. 